\title{
The regulation of infection
}

\author{
LE Nicolle MD FRCP, Editor-in-Chief
}

$\mathrm{T}_{\mathrm{c}}^{\mathrm{h}}$ he transmission of microorganisms and the severity of clinical infection are both intrinsically chaotic events. The number and complexity of variables that influence outcomes - exposure, virulence, genetics, immunity, therapeutics and health care access - together with what must surely be many 'unknown unknowns', is extraordinary. Investigators continue to address the questions: 'who gets infected?', 'who gets disease?' and 'who dies?', but current understanding supports only approximate answers for these questions. Despite this uncertainty, developed countries globally are introducing and mandating programs which, in effect, regulate infection (1-3). How should the infectious diseases physician or the medical microbiologist toiling at the Sysiphisian task of preventing and treating infections view these regulatory approaches? Will regulation subdue the fire-spouting dragon of infectious diseases, or are we just burning up resources which are better used elsewhere?

The regulation of infection has emerged in several forms. In the United States, there are requirements for public reporting of health care-acquired infections (1), which appeared hand-in-hand with 'getting to zero' as a perceived benchmark for hospital-acquired infections. Pay-for-performance regulations for reimbursement have also been introduced, including stipulating when antimicrobial therapy must be given (4) (ie, within $1 \mathrm{~h}$ for patients presenting to emergency departments with suspected pneumonia). In the United Kingdom, methicillin-resistant Staphylococcus aureus (MRSA) bacteremia rates of all health care facilities are reported centrally, and targets for bacteremia rates must be met (3). If not met, then the facility is 'retrained'. In the Netherlands, health care workers are screened after each shift during which they have contact with an MRSA patient, and if MRSA-positive, they must have eradication therapy irrespective of evidence for disease transmission (5). Persistent throat carriers who fail eradication have undergone tonsillectomy if they wished to continue working! Here in Canada, there are rumours of public reporting of hospital-acquired infections and antimicrobialresistant organisms, although the specifics are lacking.

These approaches are primarily focused on hospitalized patients and hospital-acquired infections. They have evolved from patient safety initiatives, with pressure through public advocacy - the goal is to standardize hospital practice and patient management. For the United States, the introduction of these strategies is consistent with the highly regulated approach to industry. Public health, which is not seen as an 'industry', has had a mandate to prevent infections for many years, but similar regulations have not been developed. There is no 'getting to $100 \%$ ' for immunization rates or critical incident review of each nonvaccinated child!
The initial response from the seasoned practitioner is skepticism $(1,2)$. Where is the evidence that these regulatory initiatives improve patient outcome? Are the data collection and analyses appropriate? There are always opportunities to 'game' the system. For instance, 'getting to zero' is feasible for centralline infections or ventilator-associated pneumonia in a cardiac intensive care unit in which patient-stays after surgery are seldom longer than $48 \mathrm{~h}$ to $72 \mathrm{~h}$. But is 'zero' achievable for the medical intensive care unit when intubated patients with multiple lines may stay for several weeks? In fact, these approaches may create perverse incentives - poor quality surveillance will give apparent better outcomes. Regulations requiring rapid antibiotic initiation for ill patients in the emergency department are already reported to have unintended consequences (6). A high proportion of antibiotics given to meet this strategy are inappropriate or unnecessary. This negatively impacts the parallel health care issue of antimicrobial overuse promoting antimicrobial resistance or Clostridium difficile disease.

From other perspectives, however, these regulatory initiatives may be a positive development. Interventions of documented effectiveness in preventing hospital-acquired infections have not been consistently introduced in patient care. The 'bundled approach' with monitoring of processes, and a focus on outcome measurement provides an implementation strategy that has been effective in improving practice in many facilities in which other approaches were only partially effective. 'Getting to zero' is linked to the bundle concept in which appropriate procedures are all or nothing. This approach may prove to be a durable advance in the application of infection control interventions. Systematic monitoring and observation of an event (for instance, the timing of surgical prophylaxis) increases compliance. Surveillance and reporting of outcomes, such as hospital-acquired infections, consistently leads to improvements in performance. The current extraordinary emphasis on MRSA colonization, including legislated requirements for surveillance cultures (3), seems misplaced because Staphylococcus aureus is a normal part of the human flora. On the other hand, MRSA bacteremia rates may be a surrogate for the effectiveness of infection control practices. The most effective way to decrease MRSA bacteremia, for instance, in a hospital in Great Britain, is to consistently implement strategies known to decrease any hospital-acquired bacteremia with any organism. Thus, required reporting of a single infection may have broader positive impacts for hospital-acquired infections. While the ultimate benefit and sustainability of most of these interventions remains unknown, regulatory interventions have contributed to increased awareness and, in some cases, augmented resources.

Department of Internal Medicine, University of Manitoba, Winnipeg, Manitoba

Correspondence: Dr LE Nicolle, Department of Internal Medicine, University of Manitoba, Health Sciences Centre, GG443 - 820 Sherbrook

Street, Winnipeg, Manitoba R3A 1R9. Telephone 204-787-7029, fax 204-787-4826, e-mail nicolle@cc.umanitoba.ca

Received and accepted for publication March 25, 2008 
So where is the infectious diseases physician in this? The first challenge is to remain part of the process - individuals who understand infections, infection prevention and epidemiological analysis are essential participants. The brew of politicians, public advocates, patient safety professionals and entrepreneurs who drive these approaches are seldom equipped with detailed relevant knowledge. Although many of us may not be comfortable with the political process, clinical and scientific knowledge and experience must be at the table. The role of an infectious

\section{REFERENCES}

1. Brennan PJ. In the beginning there was... heat. Infect Control Hosp Epidemiol 2006;27:329-31.

2. Weber SG, Huang SS, Oriola S, et al; Society for Healthcare Epidemiology of America; Association of Professionals in Infection Control and Epidemiology. Legislative mandates for use of active surveillance cultures to screen for methicillinresistant Staphylococcus aureus and vancomycin-resistant enterococci: Position statement from the Joint SHEA and APIC

Task Force. Infect Control Hosp Epidemiol 2007;28:249-60.

3. Johnson AP, Pearson A, Duckworth G. Surveillance and epidemiology of MRSA bacteremia in the UK. J Antimicrob Chemother 2005;56:455-62. diseases physician is to argue for programs founded on evidence; to consistently and repeatedly reaffirm the pre-eminence of knowledge-based decision-making. The second role is to identify the many remaining areas of uncertainty and articulate important residual questions. Continued advocacy for high-quality, relevant research to document the validity and effectiveness of regulatory approaches will be necessary. These messages from the infectious diseases professional will not always be heard, and often only partially understood, but our role is essential.

4. Pines JM, Hollander JE, Datner EM, Metlay JP. Pay for performance for antibiotic timing in pneumonia: Caveat emptor. Jt Comm J Qual Patient Saf 2006;32:531-5.

5. Sherman ER, Heydon KH, St John KH, et al. Administrative data fail to accurately identify cases of healthcareassociated infection. Infect Control Hosp Epidemiol 2006;27:332-7.

6. Blok HE, Troelstra A, Kamp-Hopmans TE, et al. Role of healthcare workers in outbreaks of methicillin-resistant Staphylococcus aureus: A 10-year evaluation from a Dutch university hospital. Infect Control Hosp Epidemiol 2003;24:679-85. 


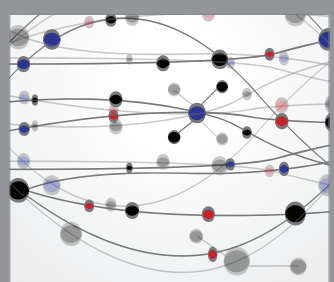

The Scientific World Journal
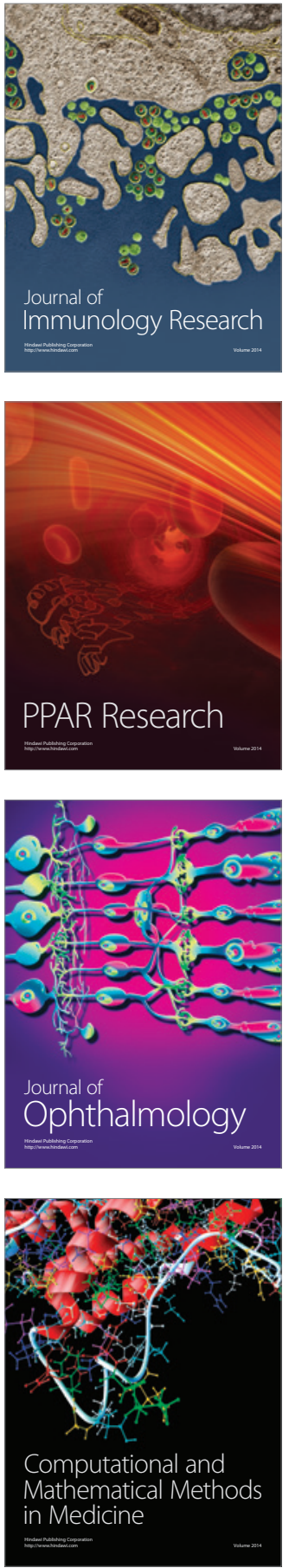

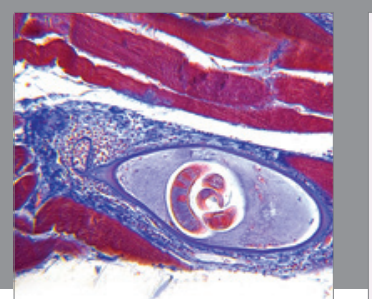

Gastroenterology Research and Practice

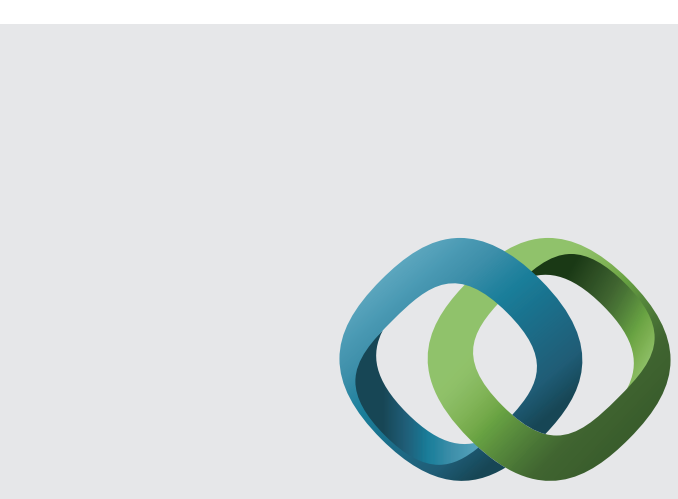

\section{Hindawi}

Submit your manuscripts at

http://www.hindawi.com
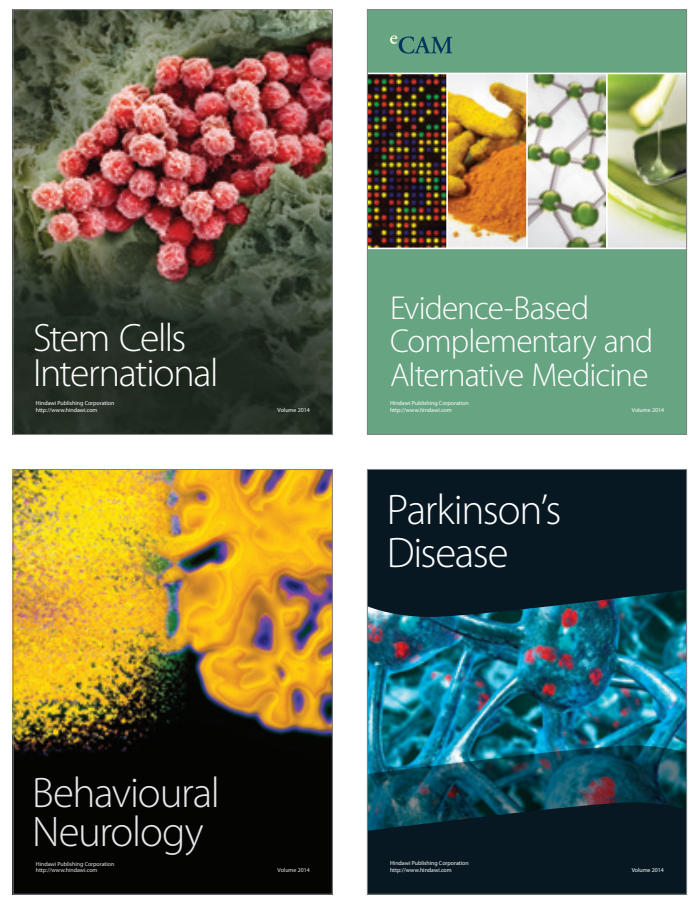
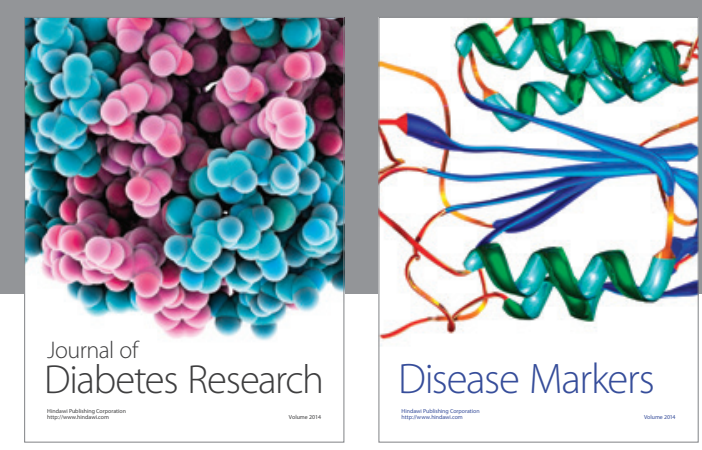

Disease Markers
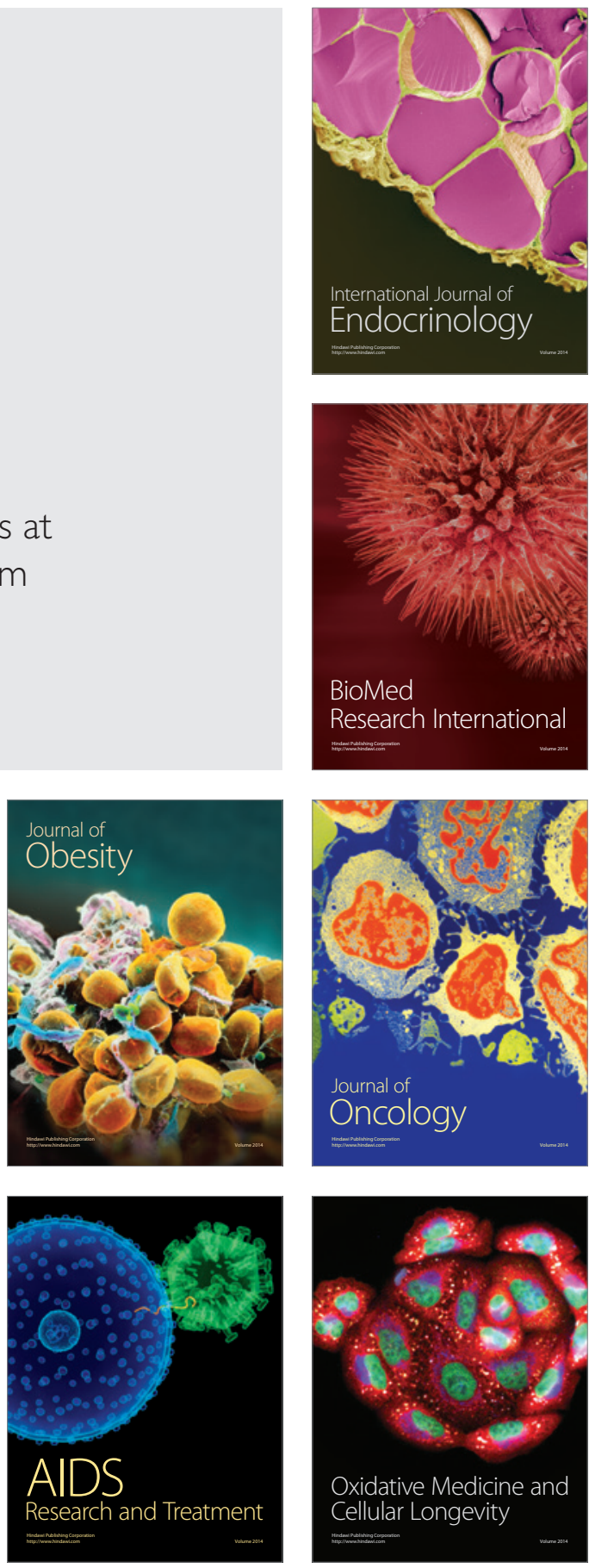